Pacific Journal of Mathematic 


\title{
SWEEDLER'S TWO-COCYCLES AND HOCHSCHILD COHOMOLOGY
}

\author{
DAVE RIFFELMACHER
}

\begin{abstract}
For any algebra $C$ over a commutative ring $k$ Sweedler defined a cohomology set which generalizes Amitsur's second cohomology group $H^{2}(C / k)$. Any Sweedler $C$-two-cocycle $\sigma$ gives rise to a change of rings functor ()$^{\sigma}$ from the category of $C$-bimodules to the category of $C^{\sigma}$-bimodules, where $C^{\sigma}$ is the $k$-algebra with multiplication altered by $\sigma$, which in turn induces a map $\phi^{n}(\sigma, M): H^{n}(C, M) \rightarrow H^{n}\left(C^{\sigma}, M^{\sigma}\right)$ on Hochschild cohomology for any $C$-bimodule $M$ and any positive integer $n$. In this paper, several properties of $\phi^{n}(\sigma, M)$ are derived, including: If $C$ is a finite dimensional algebra over a field $k, \phi^{1}(\sigma, M)$ is an injection for all $\sigma$ and $M$.
\end{abstract}

1. Introduction. In $\S 2$ we establish our notation conventions and review the basic definitions of Sweedler's two-cocycles and Hochschild cohomology. We also recall the change of rings functor ()$^{\sigma}$ associated with a Sweedler $C$-two-cycle $\sigma$ from the category of $C$-bimodules to the category of $C^{\sigma}$-bimodules for any algebra $C$ over a commutative ring $k$.

The map $\phi^{n}(\sigma, M)$ induced by ()$^{\sigma}$ from the $n$th Hochschild cohomology group $H^{n}(C, M)$ of $C$ with coefficients in the $C$-bimodule $M$ to $H^{n}\left(C^{\sigma}, M^{o}\right)$ is studied in $\S 3$. This map links the multiplicative cohomology of Sweedler and Amitsur to the additive cohomology of Hochschild. We provide an example to show that this map need not be surjective but show that if $\sigma$ is invertible in an appropriate sense $\dot{\phi}^{n}(\sigma, M)$ is actually an isomorphism. In particular, if $\sigma$ is an invertible (i.e., Amitsur) two-cocycle contained in a commutative subalgebra $A$ of $C$, then $\phi^{n}(\sigma, M)$ is an isomorphism. The behavior of $\phi^{\prime r}(\sigma, M)$ under base extension of $k$ and two-cocycles equivalent to $\sigma$ is considered, and several other results are derived which are useful in studying $\operatorname{ker} \dot{\phi}^{n}(\sigma, M)$.

In $\S 4$ we prove that if $C$ is a finite dimensional algebra over a field $k, \phi^{1}(\sigma, M)$ is injective for all $\sigma$ and $M$; that is, $\sigma$ induces an injection of the group of equivalence classes of $k$-derivations of $C$ with values in $M$ into the group of equivalence classes of $k$-derivations of $C^{\sigma}$ with values in $M^{\sigma}$. This result is compared with Flanigan's work on Gerstenhaber's deformation theory.

2. Notation and preliminaries. Let $C$ be an algebra over a commutative ring $k$ and let unadorned $\otimes$ and Hom represent $\otimes_{k}$ and $\mathrm{Hom}_{k}$, respectively. Denote the $n$-fold tensor product $C \otimes \cdots \otimes C$ 
by $C^{\otimes n}$. We denote the opposite $k$-algebra of $C$ by $C^{0}$ and call $a$ left $C \otimes C^{0}$-module a $C$-bimodule. In this section we provide a brief review of the pertinent features of Sweedler's theory of two-cocycles and Hochschild's cohomology theory.

Following Sweedler [7], we call an element $\sigma=\sum_{i} a_{i} \otimes b_{i} \otimes c_{i}$ in $C \otimes C \otimes C$ a $C$-two-cocycle if

$$
\sum_{i, j} a_{i} a_{j} \otimes b_{j} \otimes c_{j} b_{i} \otimes c_{i}=\sum_{i, j} a_{i} \otimes b_{i} a_{j} \otimes b_{j} \otimes c_{j} c_{i}
$$

and there is an element $e_{\sigma}$ in $C$ with

$$
\sum_{i} a_{i} e_{\sigma} b_{i} \otimes c_{i}=1 \otimes 1=\sum_{i} a_{i} \otimes b_{i} e_{\sigma} c_{i} .
$$

Given a $C$-two-cocycle $\sigma$ we may form a $k$-algebra $C^{\sigma}$ as follows. As an abelian group $C^{\sigma}$ is equal to $C$. For any $x$ in $C$, we use the notation $x^{\sigma}$ to indicate that we are considering $x$ as an element of $C^{\sigma}$. The product ${ }^{*}$ of any two elements $x^{\sigma}$ and $y^{\sigma}$ in $C^{\sigma}$ is defined by

$$
x^{\sigma} * y^{\sigma}=\left(\sum_{i} a_{i} x b_{i} y c_{i}\right)^{\sigma} .
$$

The $C$-two-cocycle $\sigma=\sum_{i} a_{i} \otimes b_{i} \otimes c_{i}$ is said to be cohomologous to the $C$-two-cocycle $\tau=\sum_{i} r_{i} \otimes s_{i} \otimes t_{i}$ via $\delta=\sum_{i} x_{i} \otimes y_{i}$ in $C \otimes C$ if

$$
\sum_{i, j} x_{i} a_{j} \otimes b_{j} \otimes c_{j} y_{i}=\sum_{i, j, l} r_{i} x_{j} \otimes y_{j} s_{i} x_{l} \otimes y_{l} t_{i}
$$

and

$$
\sum_{i} x_{i} e_{\sigma} y_{i}=e_{\tau}
$$

If the element $\sum_{2} x_{i} \otimes y_{i}^{0}$ in $C \otimes C^{0}$ is invertible, $\delta$ is called vertible, and $\sigma$ and $\tau$ are said to be equivalent, denoted $\sigma \sim{ }^{\circ} \tau$. In this case the $k$-algebra map $R^{\delta}: C^{\sigma} \rightarrow C^{\tau}$ defined by $R^{\delta}\left(c^{\sigma}\right)=\left(\sum_{i} x_{i} c y_{i}\right)^{\tau}$ is an isomorphism.

Definition 2.1. For any $C$-two-cocycle $\sigma=\sum_{i} a_{i} \otimes b_{i} \otimes c_{i}$, let the linear map $\phi(\sigma): C^{\sigma} \otimes C^{\sigma 0} \rightarrow C \otimes C^{0}$ be defined by

$$
x^{\sigma} \otimes y^{o^{0}} \longrightarrow \sum_{i, j} a_{i} a_{j} x b_{j} \otimes\left(c_{j} b_{i} y c_{i}\right)^{0} .
$$

$\phi(\sigma)$ is a $k$-algebra map and hence induces a change of rings functor from the category $M(C)$ of $C$-bimodules to the category $M\left(C^{\sigma}\right)$ of $C^{\sigma}$-bimodules which we will denote by ()$^{\sigma}$. The interested reader is referred to $[5,6,7]$ for more of the theory of Sweedler twococycles and the maps ()$^{\sigma}$.

Now we review the basic definition of Hochschild cohomology. 
For any integer $n \geqq 1$ and any $C$-bimodule $M$ define $\delta^{n}: \operatorname{Hom}\left(C^{\otimes n}, M\right) \rightarrow$ $\operatorname{Hom}\left(C^{\otimes(n+1)}, M\right)$ by

$$
\begin{aligned}
& (\delta f)\left(x_{1} \otimes \cdots \otimes x_{n+1}\right)=x_{1} f\left(x_{2} \otimes \cdots \otimes x_{n+1}\right) \\
& \quad+\sum_{0<i<n+1}(-1)^{i} f\left(x_{1} \otimes \cdots \otimes x_{i} x_{i+1} \otimes \cdots \otimes x_{n+1}\right) \\
& \quad+(-1)^{n+1} f\left(x_{1} \otimes \cdots \otimes x_{n}\right) x_{n+1} .
\end{aligned}
$$

The $n$th Hochschild cohomology group of $C$ with values in $M$ is then defined as $H^{n}(C, M)=\operatorname{ker} \delta^{n} / \operatorname{Im} \delta^{n-1}$. If $C$ is a projective $k$-module, it may be shown [1, Chap. IX] that $H^{n}(C, M)=\operatorname{Ext}_{c \otimes 0^{0}}^{n}(C, M)$. Further discussion of Hochschild cohomology may be found in $[1,4]$.

3. Map induced on Hochschild cohomology by a two-cocycle. The change of rings map $\phi(\sigma)$ associated with a Sweedler two-cocycle $\sigma$ introduced in Definition 2.1 induces a map $\phi^{n}(\sigma, M)$ : $H^{n}(C, M) \rightarrow H^{n}\left(C^{\sigma}, M^{\sigma}\right)$ on Hochschild cohomology which is the focus of this paper. In general, this map is not surjective, as the following example illustrates.

ExAMPLE 3.1. Let $k$ be a field and $C$ be a central separable $k$ algebra. Then $H^{n}(C, M)=\{0\}$ for all $n \geqq 1$ and all $M$. However, given any $k$-algebra $D$ with $k$-dimension of $D$ equal to the $k$-dimension of $C$, there is a $C$-two-cocycle $\tau$ with $D \simeq C^{\tau}$ [7, Theorem 6.1]. Choose $\tau$ so that $C^{\tau}=k[x] /\left\langle x^{m}\right\rangle$ with $m=k$-dimension of $C$. Then $H^{1}\left(C^{\tau}, C^{\tau}\right) \neq\{0\}$ since $d / d x$ is a nontrivial Hochschild 1-cocycle. Thus $\phi^{1}(\tau, C)$ is not surjective.

In certain cases, however, $\phi^{n}(\sigma, M)$ is actually an isomorphism.

Definition 3.2. A Sweedler $C$-two-cocycle $\sigma=\sum_{i} a_{i} \otimes b_{i} \otimes c_{i}$ is vertible if $\sum_{i, j} a_{i} a_{j} \otimes b_{j}^{0} \otimes c_{j} b_{i} \otimes c_{i}^{0}$ is invertible in $C \otimes C^{0} \otimes$ $C \otimes C^{\circ}$.

LemMA 3.3. If a Sweedler $C$-two-cocycle $\sigma$ is vertible, $\phi^{n}(\sigma, M)$ is an isomorphism for all $n$ and $M$.

Proof. Let $\sum_{l} u_{l} \otimes v_{l}^{0} \otimes w_{l} \otimes z_{l}^{0}$ be the inverse of

$$
\sum_{i, j} a_{i} a_{j} \otimes b_{j}^{0} \otimes c_{j} b_{i} \otimes c_{i}^{0} \text { and }
$$

define $\bar{\phi}(\sigma): C \otimes C^{0} \rightarrow C^{o} \otimes C^{\sigma^{0}}$ by

$$
\bar{\phi}(\sigma)\left\{\sum_{i} x_{i} \otimes y_{i}^{o}\right\}=\sum_{i, l}\left(u_{l} x_{i} v_{l}\right)^{\sigma} \otimes\left(w_{l} y_{i} z_{l}\right)^{\sigma^{0}} .
$$

Then it may be verified directly that $\bar{\phi}(\sigma)=\phi(\sigma)^{-1}$. Therefore $\phi(\sigma)$ 
is an isomorphism and hence the induced maps $\phi^{n}(\sigma, M)$ on Hochschild cohomology are isomorphisms.

Example 3.4. Let $A \leqq C$ be $k$-algebras with $A$ commutative. If $\sigma$ in $A \otimes A \otimes A \subseteq C \otimes C \otimes C$ is an invertible (i.e., Amitsur) $A$ two-cocycle, clearly $\sigma$ is a vertible $C$-two-cocycle and thus $\phi^{n}(\sigma, M)$ is an isomorphism for all $n$ and $M$.

In the next section, we will show that $\operatorname{ker}\left\{\phi^{1}(\sigma, M)\right\}=\{0\}$ for any Sweedler two-cocycle $\sigma$ if $C$ is a finite dimensional algebra over a field $k$. First, however, we need several simplifying tools. Let $\sigma$ and $\tau$ be $C$-two-cocycles such that $\sigma \sim{ }^{\delta} \tau$. Then for any $n$ and $M, \phi^{n}(\tau, M)=\underline{R}^{\delta} \circ \phi^{n}(\sigma, M)$ where $\underline{R}^{\delta}$ is induced by the algebra isomorphism $R^{\delta}: C^{\sigma} \rightarrow C^{\tau}$. Therefore we have

LEMMA 3.5. If $\sigma$ and $\tau$ are equivalent C-two-cocycles, $M$ is a $C$-bimodule and $n$ is a positive integer,

$$
\operatorname{ker}\left\{\phi^{n}(\sigma, M)\right\}=\{0\} \text { iff } \operatorname{ker}\left\{\dot{\phi}^{n}(\tau, M)\right\}=\{0\} .
$$

LEMMA 3.6. Let $S_{i}, i=1,2$, be algebras over the commutative ring $k$. Hence $C=S_{1} \times S_{2}$ is a k-algebra with diagonal k-action and canonical projections $p_{i}: C \rightarrow S_{i}, i=1,2$. If

$$
\sigma=\sum_{i}\left(a_{i 1}, a_{i 2}\right) \otimes\left(b_{i 1}, b_{i 2}\right) \otimes\left(c_{i 1}, c_{i 2}\right)
$$

is a $C$-two-cocycle, then

(i) $p_{j}(\sigma)=\sum_{i} a_{i j} \otimes b_{i j} \otimes c_{i j}$ is an $S_{j}$-two-cocycle.

(ii) If $\delta_{j}=\sum_{i} x_{i j} \otimes y_{i j}$ is a vertible element in $S_{j} \otimes S_{j}, j=1,2$, then

$$
\begin{aligned}
\hat{o}= & (1,0) \otimes(0,1)+(0,1) \otimes(1,0) \\
& +\sum_{i}\left(x_{i 1}, 0\right) \otimes\left(y_{i 1}, 0\right) \\
& +\sum_{\imath}\left(0, x_{i 2}\right) \otimes\left(0, y_{i 2}\right)
\end{aligned}
$$

is a vertible element in $C \otimes C$ and $\sigma \sim{ }^{\circ} \tau$ defines a C-two-cocycle $\tau$ with $e_{\tau}=\left(\sum_{i} x_{i 1} e_{\sigma_{1}} y_{i 1}, \sum_{i} x_{i 2} e_{\sigma_{2}} y_{i 2}\right)$ if $e_{\sigma}=\left(e_{\sigma_{1}}, e_{\sigma_{2}}\right)$.

Proof.

(i) follows trivially since an algebra map clearly preserves the two-cocycle relations. that

(ii) If $\bar{\delta}_{j}=\sum_{i} \bar{x}_{i j} \otimes \bar{y}_{i j}$ is the verse of $\delta_{j}$, then one may show

$$
\begin{aligned}
\bar{\delta}= & (1,0) \otimes(0,1)+(0,1) \otimes(1,0) \\
& +\sum_{i}\left(\bar{x}_{i 1}, 0\right) \otimes\left(\bar{y}_{i 1}, 0\right)
\end{aligned}
$$




$$
+\sum_{i}\left(0, \bar{x}_{i 2}\right) \otimes\left(0, \bar{y}_{i 2}\right)
$$

is the verse of $\delta$. The form of $e_{\tau}$ is clear.

Suppose now that $k$ is a field and let $L$ be an extension field of $k$. The natural injection $C \rightarrow C \otimes L$ induces a map $C \otimes C \otimes C \rightarrow$ $(C \otimes L) \otimes_{L}(C \otimes L) \otimes_{L}(C \otimes L)$. Let $\sigma \otimes 1$ denote the image of the $C$-two-cocycle $\sigma$ under this map. Then we have a commutative diagram with exact rows

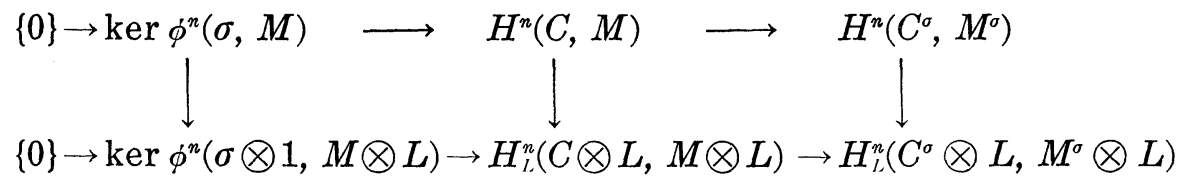

where $H_{L}^{n}(-,-)$ denotes the Hochschild cohomology as an $L$-algebra rather than as a $k$-algebra. Since $L$ is a field extension of $k$, it follows easily by linear algebra from the definition of Hochschild cohomology (cf. $\S 2)$, noting $(C \otimes L) \otimes_{L} n \simeq\left(C^{\otimes n}\right) \otimes L$, that the map $H^{n}(C, M) \rightarrow H_{L}^{n}(C \otimes L, M \otimes L)$ is injective. Thus the above commutative diagram allows one to conclude that $\operatorname{ker} \phi^{n}(\sigma \otimes 1, M \otimes L)=\{0\}$ implies that $\operatorname{ker} \phi^{n}(\sigma, M)=\{0\}$.

LEMMA 3.7. If $C$ is an algebra over an algebraically closed field $k$ which is Artinian as a ring and $\sigma$ is a C-two-cocycle, there is a C-two-cocycle $\tau$ with $\sigma \sim{ }^{i} \tau$ and $e_{\tau}=1$.

Proof. Since $C$ is Artinian, the Jacobson radical $J(C)$ is nilpotent and thus by [5, Theorem 4.7] $\sigma \sim q(\tau)$ where $q$ is the endomorphism of $C$ induced by projection modulo $J(C)$. (Note that since $k$ is algebraically closed, the semisimple algebra $C / J(C)$ is actually separable over k.) We may henceforth assume $C$ is semisimple and thus by Wedderburn-Artin structure theory we have $C=\prod_{i=1}^{m} S_{i}$ where $S_{i}=M\left(n_{i}, k\right)$, the algebra of $n_{i}$ by $n_{i}$ matrices over $k$. Then by Lemma 3.6 it is sufficient to show that the $S_{i}$-two-cocycle $p_{i}(\sigma)$ is equivalent via $\hat{o}_{i}$ to an $S_{i}$-two-cocycle $\tau_{i}$ with $e_{\tau_{i}}=1$. If $n_{i}=1$ and $S_{i}=k$, one may take $\delta_{i}=1 \otimes e_{o_{i}}$ (cf. $\left.[6, \S 1]\right)$. If $n_{i}>1$, then the existence of $\delta_{i}=\sum_{j} x_{j i} \otimes y_{j i}$ with $e_{\sigma_{i}} \sim{ }^{\hat{\delta}_{i}} e_{\tau_{i}}$ and $\sum_{j} x_{j i} e_{\sigma_{i}} y_{j i}=1$ is assured by [7, Theorem 6.1].

4. Injectivity of $\phi^{1}(\sigma, M)$. In this section we prove

THEOREm 4.1. Let $C$ be a finite dimensional algebra over a field $k, \sigma$ be a Sweedler C-two-cocycle, and $M$ be a C-bimodule. Then $\phi^{1}(\sigma, M): H^{1}(C, M) \rightarrow H^{1}\left(C^{\sigma}, M^{\sigma}\right)$ is injective. 
In light of the results in $\S 3$, we need only to prove:

THEOREM 4.2. If $k$ is an algebraically closed field, $C$ is a finite dimensional k-algebra, $\sigma$ is a Sweedler $C$-two-cocycle, and $M$ is a $C$-bimodule, then there is a Sweedler C-two-cocycle $\tau$ with $\sigma$ equivalent to $\tau$ and $\phi^{1}(\tau, M)$ injective.

To establish this theorem we need several preliminary results.

Lemma 4.3. If $k$ is a field, $C$ is a k-algebra, $\sigma$ is a Sweedler $C$-two-cocycle, and $M$ is a C-bimodule, the map $\phi^{1}(\sigma, M)$ is induced by the map

$$
d \longrightarrow\left(x^{\sigma} \longrightarrow\left(\sum_{i, j} a_{i} a_{j} e_{o} d\left(b_{j} x b_{i} e_{\sigma}\right) c_{i} c_{j}\right)^{\sigma}\right)
$$

from the group $\operatorname{Der}_{k}(C, M)$ of k-derivations of $C$ with values in $M$ to $\operatorname{Der}_{k}\left(C^{\sigma}, M^{\sigma}\right)$.

Proof. This lemma may be proved using the definition of $\phi(\sigma)$.

LEMMA 4.4. Let $C$ be an algebra over the commutative ring $k$, $M$ be a $C$-bimodule, and $d: C \rightarrow M$ be a k-derivation. Let $\alpha=\sum_{i} a_{i} \otimes$ $b_{i} \otimes c_{i}$ and $m$ be elements of $C \otimes C \otimes C$ and $M$, respectively, such that

$$
\begin{aligned}
& d(x)=\sum_{i} a_{i} x b_{i} m c_{i}-\sum_{i} a_{i} m b_{i} x c_{i} \equiv[x, m]_{\alpha} \\
& d\left(b_{i}\right)=0 \text { for all } i \\
& \sum_{i} a_{i} b_{i} \otimes c_{i}=1 \otimes 1=\sum_{i} a_{i} \otimes b_{i} c_{\imath} .
\end{aligned}
$$

Then $d(x)=x m-m x=[x, m]$ for all $x$ in $C$.

Proof. Letting [,] denote the usual Lie bracket, we have

$$
\begin{aligned}
d(x) & =\sum_{i} a_{i} x b_{i} m c_{i}-\sum_{i} a_{i} m b_{i} x c_{i} \\
& =\sum_{i} a_{i} x\left[b_{i}, m\right] c_{i}+\sum_{i} a_{i}\left[b_{i}, m\right] x c_{i}+[x, m] .
\end{aligned}
$$

Since $d\left(b_{i}\right)=0$ for all $i$,

$$
\left[b_{i}, m\right]=-\sum_{j} a_{j} b_{i}\left[b_{j}, m\right] c_{j}-\sum_{j} a_{j}\left[b_{j}, m\right] b_{i} c_{j} .
$$

Hence we may rewrite eqn. (4.5) as

$$
d(x)=-\sum_{i j} a_{i} x a_{j} b_{i}\left[b_{j}, m\right] c_{j} c_{i}
$$




$$
\begin{aligned}
& -\sum_{i, j} a_{i} x a_{j}\left[b_{j}, m\right] b_{i} c_{j} c_{i} \\
& -\sum_{i, j} a_{i} a_{j} b_{i}\left[b_{j}, m\right] c_{j} x c_{i} \\
& -\sum_{i, j} a_{i} a_{j}\left[b_{j}, m\right] b_{i} c_{j} x c_{i}+[x, m] \\
= & -\sum_{i} a_{i} x d\left(b_{i}\right) c_{i}-\sum_{i} a_{i} d\left(b_{i}\right) x c_{i}+[x, m] \\
d(x)= & {[x, m] }
\end{aligned}
$$

and we are done.

Now we are ready to prove Theorem 4.2:

Since we are assuming $C$ is a finite dimensional algebra over an algebraically closed field, we may write $C=B \oplus J(C)$ with $J(C)$ the Jacobson radical of $C$ and $B$ a $k$-separable subalgebra of $C$. By [5, Theorem 4.7], $\sigma$ is equivalent to its projection modulo $J(C)$, a $C$-twococycle $\sigma_{1}$ in $B \otimes B \otimes B$. Then using Lemma 4.3 and the fact that $H^{1}(B, M)=\{0\}$ [4, Theorem 4.1], the map $\operatorname{Der}_{k}(C, M) \rightarrow \operatorname{Der}_{k}\left(C^{\sigma_{1}}, M^{\sigma_{1}}\right)$ which induces $\phi^{1}\left(\sigma_{1}, M\right)$ is given by $d \rightarrow\left(x^{\sigma_{1}} \rightarrow d(x)^{\sigma_{1}}\right)$. Thus $\phi^{1}\left(\sigma_{1}, M\right)$ will be injective if for any $m$ in $M, d\left(x^{\sigma_{2}}\right)=x^{\sigma_{2}} * m^{\sigma_{2}}-m^{\sigma_{2}} * x^{\sigma_{2}}$ for all $x$ implies that $d(x)=x m-m x$ for some two-cocycle $\sigma_{2}$ equivalent to $\sigma_{1}$. This follows from Lemma 3.7 and Lemma 4.4 .

REMARK 4.5. Theorem 4.2 may be paraphased as "multiplication alteration shrinks the separable part of $C$." Since $J(C)^{\sigma} \subseteq J\left(C^{\sigma}\right)$ for $J(C)$ nilpotent [5, Lemma 2.1], multiplication alteration adds to the nilpotency of $C$. Hence the effect of multiplication alteration is in a sense opposite to the effect of Gerstenhaber's deformation theory [3] which adds to the separable part of $C$ and shrinks the radical [2, Theorem 1].

ACKNOWLEDGment. I wish to thank Moss Sweedler for discussions during which the proof of Lemma 4.4 evolved.

\section{REFERENCES}

1. H. Cartan and S. Eilenberg, Homological Algebra, Princeton University Press, 1956.

2. F. Flanigan, Straightening-out and semirigidity in associative algebras, Trans. Amer. Math. Soc., 138 (1969), 415-425.

3. M. Gerstenhaber, On the deformation of rings and algebras, Ann. of Math., 79 (1964), 59-103.

4. G. Hochschild, On the cohomology groups of an associative algebra, Ann. of Math., 46 (1945), 58-67.

5. D. Riffelmacher, Multiplication alteration and related rigidity properties of algebras, Pacific J. Math., 71 (1977), 139-157.

6. - Sweedler's two-cocycles and generalizations of theorems on Amitsur 
cohomology, Trans. Amer. Math. Soc., 251 (1979), 255-265.

7. M. Sweedler, Multiplication alteration by two-cocycles, Illinois J. Math., 15 (1971), $302-323$.

Received March 29, 1979.

Bell Laboratories

HOLMDEL, NJ 07733 


\section{PACIFIC JOURNAL OF MATHEMATICS}

\section{EDITORS}

DONALD BABBITT (Managing Editor)

University of Galifornia

Los Angeles, California 90024

Hugo RossI

University of Utah

Salt Lake City, UT 84112

C. C. MoOre AND ANDrew OGG

University of California

Berkeley, CA 94720

\section{J. DugundjI}

Department of Mathematics University of Southern California

Los Angeles, California 90007

R. Finn and J. Milgram

Stanford University

Stanford, California 94305

ASSOCIATE EDITORS

E. F. Beckenbachi

B. H. NeumanN

F. WOLF

K. YOSHIDA

\section{SUPPORTING INSTITUTIONS}

UNIVERSITY OF BRITISH COLUMBIA

CALIFORNIA INSTITUTE OF TECHNOLOGY

UNIVERSITY OF CALIFORNIA

MONTANA STATE UNIVERSITY

UNIVERSITY OF NEVADA, RENO

NEW MEXICO STATE UNIVERSITY

OREGON STATE UNIVERSITY

UNIVERSITY OF OREGON
UNIVERSITY OF SOUTHERN CALIFONIA

STANFORD UNIVERSITY

UNIVERSITY OF HAWAII

UNIVERSITY OF TOKYO

UNIVERSITY OF UTAH

WASHINGTON STATE UNIVERSITY

UNIVERSITY OF WASHINGTON

Printed in Japan by International Academic Printing Co., Ltd., Tokyo, Japan 


\section{Pacific Journal of Mathematics \\ Vol. 89 , No. 1 \\ May, 1980}

David Bressoud, A note on gap-frequency partitions ................ 1

John David Brillhart, A double inversion formula ................. 7

Frank Richard Deutsch, Günther Nürnberger and Ivan Singer, Weak

Chebyshev subspaces and alternation .......................

Edward Richard Fadell, The relationship between Ljusternik-Schnirelman

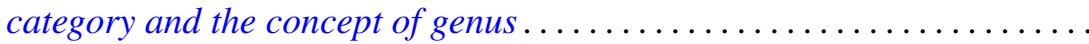

Harriet Jane Fell, On the zeros of convex combinations of polynomials.......

John Albert Fridy, An addendum to: "Tauberian theorems via block

dominated matrices" ..................................

Andrzej Granas, Ronald Bernard Guenther and John Walter Lee, Applications of topological transversality to differential equations. I. Some nonlinear

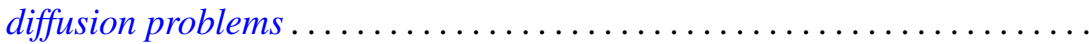

David E. Handelman and G. Renault, Actions of finite groups on self-injective

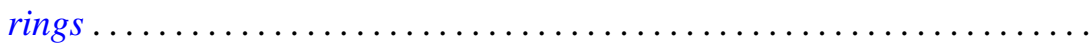

Michael Frank Hutchinson, Local $\Lambda$ sets for profinite groups .............

Arnold Samuel Kas, On the handlebody decomposition associated to a

Lefschetz fibration...

Hans Keller, On the lattice of all closed subspaces of a Hermitian space.....

P. S. Kenderov, Dense strong continuity of pointwise continuous

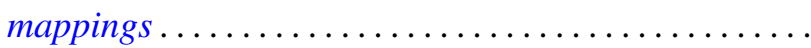

Robert Edward Kennedy, Krull rings.................

Jean Ann Larson, Richard Joseph Laver and George Frank McNulty,

Square-free and cube-free colorings of the ordinals ...

Viktor Losert and Harald Rindler, Cyclic vectors for $L^{p}(G)$

John Rowlay Martin and Edward D. Tymchatyn, Fixed point sets of

1-dimensional Peano continua...

Augusto Nobile, On equisingular families of isolated singularities ...

Kenneth Joseph Prevot, Imbedding smooth involutions in trivial bundles ...

Thomas Munro Price, Spanning surfaces for projective planes in four space.

Dave Riffelmacher, Sweedler's two-cocycles and Hochschild cohomology....

Niels Schwartz, Archimedean lattice-ordered fields that are algebraic over

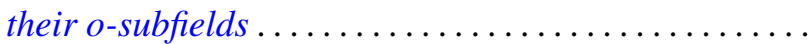

Chao-Liang Shen, A note on the automorphism groups of simple dimension groups.

Kenneth Barry Stolarsky, Mapping properties, growth, and uniqueness of

Vieta (infinite cosine) products ...

Warren James Wong, Maps on simple algebras preserving zero products. I.

The associative case ............................. 